\title{
La investigación como estrategia pedagógica para la convivencia escolar ${ }^{1}$
}

\section{Research as a pedagogical strategy for school coexistence}

DOI: http://dx.doi.org/10.17981/cultedusoc.9.1.2018.09

Fecha de recepción: 18/04/2018. Fecha de aceptación: 17/07/2018

Fray Alexander García-Martínez ${ }^{2}$

Gladys María Peña-Orozco ${ }^{3}$

Para citar este artículo

García-Martínez, F. y Peña-Orozco, G. (2018). La investigación como estrategia pedagógica para la convivencia escolar. Cultura. Educación y Sociedad 9(1), 121-133. DOI: http://dx.doi.org/10.17981/cultedusoc.9.1.2018.09

\section{Resumen}

La investigación como estrategia pedagógica es una metodología educativa que permite dirigir un aprendizaje en contexto, experimentando formas y estilos para la gestión de conocimiento mediada por momentos reflexivos, potenciando el desarrollo de competencias. El estudio buscó utilizar la investigación como estrategia pedagógica para la convivencia escolar. Estuvo guiado bajo el método cualitativo, desde el modelo de investigación acción, con un diseño descriptivo, la población estuvo conformada por cuarenta (40) estudiantes del grado sexto de la Institución Educativa Buenos Aires, de Aracataca - Magdalena. Se utilizaron técnicas de recolección como: relatoría individual e institucional y diario de campo. Los resultados muestran que, en la institución se observa un deterioro en la convivencia escolar, por ello se hizo necesario fortalecer valores, para la formación personal, que se refleje en sus relaciones personales, orientados a propiciar una educación integral, democrática, generando espacios de participación activa, mediante una cultura de paz.

Palabras clave: investigación como estrategia pedagógica, convivencia escolar, educación básica.

\begin{abstract}
Research as a pedagogical strategy is an educational methodology that allows directing a learning in context, experimenting forms and styles for the management of knowledge mediated by reflexive moments, promoting the development of competences. The study sought to use research as a pedagogical strategy for school coexistence. It was guided under the qualitative method, from the model of action research, with a descriptive design, the population was conformed by forty (40) students of the sixth grade of the Educational Institution Buenos Aires, of Aracataca - Magdalena. Collection techniques were used such as: individual and institutional rapporteur and field journal. The results show that, in the institution, a deterioration in the school coexistence is observed, for that reason it was necessary to strengthen values, for the personal formation, that is reflected in their personal relationships, oriented to propitiate an integral, democratic education, generating spaces of active participation, through a culture of peace.
\end{abstract}

Keywords: research as a pedagogical strategy, school cohabitation, basic education.

\footnotetext{
${ }^{1}$ Este artículo ha sido derivado del Programa de Fortalecimiento de la Cultura Ciudadana y Democrática CT+I a través de la IEP apoyada en TIC en el Departamento de Magdalena: CICLON

${ }^{2}$ Docente de la Institución Educativa Departamental Buenos Aires y líder del grupo de investigación observatorio de convivencia escolar.Correo de correspondencia: fraygarcia2112@hotmail.com

${ }^{3}$ Docente de la Institución Educativa Buenos Aires, Sede principal y miembro del grupo de investigación observatorio de convivencia escolar.
}

- The author; licensee Universidad de la Costa - CUC. 


\section{Introducción}

La investigación en el contexto educativo a todo nivel de formación ha encontrado un espacio privilegiando dinamizando los procesos de formación, y es por ello que año tras año ha tomado fuerza un enfoque particular que aporta a la construcción de conocimientos capaz de responder a las exigencias de la cotidianidad escolar, así como de su contextualización en tiempos, favoreciendo la construcción de una convivencia escolar.

La trayectoria didáctica del enfoque investigador se remonta a los albores de la Escuela Nueva europea y de la Escuela Progresista americana y ha continuado a lo largo del siglo XX. Los trabajos de autores como Dewey, Decroly, Piaget, Freinet, Carr y Kemmis, Stenhouse, Shemilt, entre otros, proporcionaron las bases psico-socio-pedagógicas de este enfoque. Unas bases que posteriormente han ido evolucionando y desarrollándose con los nuevos conocimientos proporcionados por las ciencias de la educación.

Cabe decir que, partiendo de la capacidad innata del ser humano para sentir curiosidad, explorar, conocer, razonar y transformar la realidad, las propuestas de investigación escolar incorporan este potencial cognitivo a las aulas y promueven que los alumnos y maestros indaguen, averigüen, curioseen, examinen, interroguen, exploren, estudien y comprendan cuestiones y problemáticas interesantes para ambos con la determinación de reconstruir sus conocimientos.

Plantear el proceso de enseñanza y aprendizaje en función de una dinámica basada en la investigación escolar es una de las propuestas más reiteradas en la tradición innovadora en educación. Desde las primeras propuestas en este sentido se ha venido planteando organi- zar los procesos del aula en función de asuntos que despierten el interés de los estudiantes, incorporando interrogantes y propuestas de acción que les involucren activamente.

Ante los cambios acelerados de conocimiento y la diversidad de paradigmas, se requiere de maestros y maestras competentes que den respuesta a los problemas de una realidad compleja y dinámica; que adopten una actitud reflexiva y crítica con respecto a la realidad educativa y que posean idoneidad técnico-profesional para investigar científicamente esa realidad y transformarla creativamente. En el campo educativo, como en el resto de las ciencias, la investigación se ha constituido en una actividad precisa y elemental.

Por este motivo, se ha originado la investigación educativa, como disciplina que trata las cuestiones y problemas relativos a la naturaleza, epistemología, metodología, fines y objetivos en el marco de la búsqueda progresiva de conocimiento en el ámbito educativo. El concepto de investigación educativa se ha ido modificando a medida que han surgido nuevos enfoques para el tratamiento de los fenómenos educativos. Actualmente, son variados los significados atribuidos a la expresión investigación educativa, dependiendo de la diversidad de objetivos y características que se le establecen.

En efecto, la investigación educativa presenta una serie de características particulares. La combinación de los objetivos y fines que pretende, la particularidad de los fenómenos que estudia y la colectividad de los métodos que emplea son dimensiones que le otorgan especificidad propia a la vez que hacen más compleja y ardua su descripción y estudio.

Dicho en otras palabras, se considera que el método es un modo de abordar los 
problemas, siendo la naturaleza del objeto a estudiar, la que hace recomendables posibles métodos y técnicas específicas. El deseo de utilizar un solo método para la aplicación de todos los fenómenos, tanto naturales como sociales, es sólo eso, un simple deseo. La realidad educativa es dinámica, interactiva y compleja, está conformada por aspectos éticos, morales, políticos y sociales que se prestan más a su examen desde planteamientos humanístico-interpretativos sin que ello implique el abandono de técnicas e instrumentos de carácter cuantitativo, de allí el grado de significancia de la investigación como estrategia pedagógica (IEP).

Uno de los elementos auto observables de la IEP, son las estrategias de autoformación, formación de saber y conocimiento; y apropiación para maestros(as) acompañantes investigadores y coinvestigadores, fortaleciendo la apropiación social y conformación de comunidades, en búsqueda de la convivencia escolar, desarrollando en estos competencias tecnológicas, científicas, comunicativas, pedagógicas y de gestión curricular que impactan el perfil del docente, buscando otra mirada hacia la investigación social, lo que humanizara cada plan de asignatura que se imparte en los espacios de aprendizajes significativos.

En este sentido la IEP, desarrolla un proceso sistemático, dinámico, práctico y creativo, ligada a la acción cotidiana de los centros a sus contextos y propias realidades, es por ello fundamental formar a los profesionales de la educación para su capacitación en actividades de investigación, que les permita reflexionar sobre su práctica educativa y actuar en consecuencia. El trabajo diario de los docentes debe ser basado en estrategias que logren en la medida el crecimiento personal de los estudiantes. La enseñanza no debe ser mecánica, debe estar abierta a cambios y a una pedagogía que genere experiencias enriquecedoras, tanto para el docente como para los estudiantes.

Se pretende con estos espacios de formación y reflexión que el docente logre integrar la IEP en sus currículos, como medio para alcanzar los propósitos educativos y pedagógicos planteados en los PEI, a partir del trabajo colaborativo y el diálogo de saberes y la negociación cultural. Por ello se retoma el proyecto de investigación de aula como el elemento que va hacer real la incorporación de la investigación como estrategia a los procesos de enseñanza y aprendizaje.

Estos se complementan con espacios de apropiación para que el maestro/a intercambie experiencias y enriquezca su práctica pedagógica a partir de la conformación de redes y comunidades de saber y práctica, donde se propicia un diálogo de saberes, se compare y retroalimenta la experiencia de acompañamiento y coinvestigación de los proyectos de investigación en el aula. Por su parte, el docente investigador pasa a otra instancia en donde a partir de la reflexión de su práctica identifica temáticas de investigación con el fin de profundizar y producir nuevo conocimiento.

En la actualidad la educación ha presentado avances significativos mediante la integración de tecnologías, cultura, ciudadanía e innovación a los contenidos curriculares, para esto los docentes han cambiado su rol autoritario propuesto por la educación tradicional, implementando modelos pedagógicos que permitan una interacción entre docentes y estudiantes, con este estudio se buscó fomentar la convivencia escolar mediante la Investigación como estrategia pedagógica en la Institución Educativa Departamental de Buenos Aires. 
La falta de una educación encaminada al fortalecimiento de valores ha dejado en la sociedad actual muchas desventajas, dificultades y espacios vacíos en el aprendizaje de los estudiantes. Estas debilidades responden a muchos factores tales como: insuficiencia de autoridad y función tutelar de los padres, la falta de concientización por parte de los educandos en crear una sociedad respetuosa tolerante y humanizante.

$\mathrm{Al}$ analizar la convivencia de los estudiantes del grado séptimo de la Institución Educativa departamental de Buenos Aires, se vivencian problemas de aceptación, irrespeto a sus semejantes, y falta de tolerancia por sus compañeros; en consecuencia, la distracción es mayor viéndose afectado el aprendizaje, porque los estudiantes pierden la concentración, actitud de aprender y participar activamente en el desarrollo de las actividades pedagógicas. Otro aspecto importante que vale la pena mencionar, es la falta de conciencia por el mal comportamiento y hasta qué punto, éste afecta a los demás; se evidencia la ausencia de una reflexión personal acerca del comportamiento y las repercusiones que tiene en el aspecto personal y social.

En la institución se observa un deterioro en la convivencia escolar, esto influye en todos los entorno de la vida de estudiantes, convirtiéndose en una preocupación de los entes educativos, por ello se hace necesario el fortalecer los valores, para que sean adquiridos de manera eficaz en la formación personal, de tal manera que se reflejen en su relación con sus semejantes y con el entorno, orientados a propiciar una educación integral, democrática, generando espacios de participación activa, mediante una cultura de paz.

Por tal motivo al utilizar la investigación como estrategia pedagógica para la convivencia escolar en educación básica, se busca

\section{La convivencia escolar mediante la} IEP: praxis en la educación básica

Cabe resaltar que, la convivencia escolar se trata de la construcción de un proceder de relación entre las personas de una colectividad, sustentada en la obediencia y en la solidaridad recíproca, expresada en la interrelación armoniosa y sin intimidación entre los diferentes actores y estamentos de la comunidad educativa. Tiene un enfoque eminentemente formativo. Se deben enseñar y aprender una suma de conocimientos, habilidades y valores que permiten poner en práctica el vivir en paz y armonía con otros, porque es la base para el ejercicio de la ciudadanía. En la escolaridad, estos aprendizajes están establecidos tanto en los objetivos fundamentales transversales como en los objetivos fundamentales verticales.

La convivencia escolar implica el desarrollo de habilidades interpersonales armoniosas, pero también, implica objetivos comunes en torno a comportamientos no violentos y de igualdad entre hombres y mujeres, lo cual se puede generar a través de talleres de prevención de la violencia escolar con perspectiva de género. (Mendoza, 2015)

Por tal motivo, se considera enaltecer los aprendizajes con una adecuada convivencia en la escuela, como un factor necesario para transformar positivamente la educación e incidir de manera favorablemente en el logro de los aprendizajes. Por tanto, es importante considerar las necesidades e intereses de los estudiantes para ayudar al desarrollo de una civilización conviviente, inclusiva y respetuosa de la diversidad. Así mismo, se debe instalar, mejorar y consolidad prácticas y estrategias que permitan cumplir con los objetivos propuestos y con ellos mejorar la calidad de educación impartida en un clima que propicia el desarrollo integral de los estudiantes. 
En otras palabras, el enfoque formativo contiene una dimensión preventiva, expresada en el progreso de conocimientos, habilidades y actitudes que permitan formar personas autónomas, capaces de tomar decisiones personales y de anticiparse a las situaciones que amenazan o alteran el aprendizaje de la convivencia, cautelando en todo momento el proceso didáctico y las estrategias pedagógicas. En este sentido, la dimensión preventiva implica superar la noción de riesgo y no se limita a informar o prohibir, sino que apunta a formar para actuar con anticipación. Al abordar la convivencia escolar mediante la IEP, su praxis con sus actores, en la educación básica permite un procesos sistémico y holístico en el quehacer educativo, utilizando las sesiones de aprendizaje como un centro donde se socialice espacios colaborativos de auto aprendizaje, (ver tabla 1).
La convivencia se entiende como un medio de interacción social, que debe ser enfrentado a diario por el ser humano, donde usualmente debe compartir criterios de acuerdo con su proceso de desarrollo. Etimológicamente, el término convivencia proviene de conviviere, que significa vivir en compañía de otros, es decir, cohabitar. Convivir hace parte del reconocimiento de que quienes comparten por diversos motivos, escenarios y actividades dentro de un mismo sistema, sujetos a normas que buscan favorecer la interacción, prevenir los conflictos y consecuencias. (López, Carvajal, Soto \& Urrea, 2013).

Según la Ley 1620 de 2013, la convivencia escolar se entiende como la acción de vivir en compañía de otras personas en el contexto escolar de manera pacífica y armónica. Esta hace referencia al conjunto de relaciones que ocurren entre

Tabla 1

Sistematización de la convivencia escolar mediante la IEP y su praxis en la educación básica.

\begin{tabular}{cl}
\hline $\begin{array}{c}\text { Convivencia escolar } \\
\text { mediante la IEP }\end{array}$ & \multicolumn{1}{c}{ Praxis en la educación básica } \\
\hline & $\begin{array}{l}\text { Afianzar la comprensión de la dimensión formativa de la convivencia escolar en } \\
\text { todo el sistema educativo, y resituarla como el ingrediente central de la gestión } \\
\text { institucional. }\end{array}$ \\
\cline { 2 - 2 } & $\begin{array}{l}\text { Fortalecer la enseñanza de los conocimientos, habilidades, actitudes y valores } \\
\text { propuestos en los Objetivos transversales, como los aprendizajes básicos para el } \\
\text { ejercicio de la convivencia escolar. }\end{array}$ \\
\cline { 2 - 3 } $\begin{array}{c}\text { Sistematización auto } \\
\text { observable ente: } \\
\text { el alumno, profesor y } \\
\text { comunidad. }\end{array}$ & $\begin{array}{l}\text { Promover el compromiso y la participación de la comunidad educativa, en la } \\
\text { la convivencia escolar, y el ejercicio de los derechos y deberes de cada uno de los } \\
\text { actores. }\end{array}$ \\
\cline { 2 - 2 } & $\begin{array}{l}\text { Fomentar en todos los actores sociales y de la comunidad educativa, una } \\
\text { comprensión compartida de la prevención, la resolución de conflictos y } \\
\text { la violencia escolar, incluido el acoso sistemático, desde una perspectiva } \\
\text { formativa. }\end{array}$ \\
\cline { 2 - 3 } & $\begin{array}{l}\text { Promover una comprensión formativa de la convivencia escolar en las } \\
\text { estrategias y acciones preventivas que implementa el interceptor u otras } \\
\text { instituciones en las comunidades educativas. }\end{array}$ \\
\hline
\end{tabular}


las personas que hacen parte de la comunidad educativa, esto debe enfocarse al logro de objetivos académicos y el desarrollo integral. Así mismo, la presente Ley afirma que el clima escolar positivo no se improvisa, ni se da de forma autónoma, esta debe construirse mediante espacios de participación donde los estudiantes tengan voz en las decisiones institucionales.

Es así como surge, en la época moderna, la preocupación por una protección integral a los estudiantes, observándolos como sujetos potenciales en nuevas acciones de política legislativa en casi todos los países del mundo, lo que ha generado la consagración de los derechos, a través de preceptos, valores y principios constitucionales, generando un análisis científico. (Herrera, 2013)

Es importante señalar que, convivencia y conflicto se presentan en un mismo escenario. Esto se debe a que existen diferencias entre pensamientos, ideologías, actitudes y comportamientos, donde no se puede omitir que en la sociedad actual los conflictos algunas veces, se resuelven violentamente y aunque las situaciones violentas aparezcan de forma aislada, no se puede negar su existencia. Ahora bien, los medios de comunicación han venido presentando en los últimos años un sin número de noticias asociadas a la violencia escolar, generando una gran preocupación a nivel Nacional, por tal razón desde la academia se han propuesto diversas estrategias para mitigar dicha problemática, (Caballero, 2010).

Partiendo de lo anterior, es necesario observar los conflictos escolares como una situación normal, propia de la interacción en el ámbito escolar, esta ha existido siempre y no se debe enfocar al tratamiento ortodoxo y autoritario, por el contrario, los entes educativos deben proponer diversas estrategias innovadoras que propendan una cultura de paz, mediante la construcción de espacios equitativos e igualitarios para las personas que conforman la comunidad educativa.

En el conjunto global de relaciones interpersonales que se producen en un centro educativo, son importantes, ya que, los propios estudiantes establecen entre sí. Sin embargo, es necesario aprender a observarlas, desde una perspectiva dialéctica y no estática. Si se considera que las relaciones personales se desenvuelven en una dinámica compleja, que hace depender a unos sistemas de otros, las relaciones que van desplegando los alumnos/as entre sí, se denominan microsistema de los iguales. El microsistema de los iguales, cuando se estabiliza como consecuencia de la constancia temporal de las experiencias diarias, se configura como un ámbito humano, que proporciona a los jóvenes pautas para organizar su comportamiento social, aportándoles indicadores sobre lo que es prudente hacer o no en un contexto determinado, Ortega. (2015).

En ese sentido la escuela se convierte en la mejor institución para generar una formación integral basada en la paz y los derechos humanos, sin embargo, esta no se encuentra aislada del entorno social, al contrario, es el lugar donde convergen todos los agentes de la comunidad. En realidad, los fenómenos de violencia escolar se han producido siempre, quizás con la misma intensidad. No obstante, ahora se han visibilizado más porque afectan a más personas, y porque los medios de comunicación, los padres y madres de los alumnos y la sociedad en general, se han sensibilizado frente a las problemáticas relacionadas con la educación, Colombo (2011). 
La educación para la paz y la convivencia debe servir como elemento integrador transversal a las asignaturas. Desde esta perspectiva, se deben incorporar en el plan de aula de los maestros acciones que fomenten una cultura de paz y sana convivencia, fortaleciéndolas en actividades específicas y métodos generales de su especialidad, ampliando el sentido educativo de la misma. Por lo cual los temas transversales introducen nuevos contenidos en el currículo, pero sobre todo reformulan y reintegran los existentes desde un nuevo enfoque integrador e interdisciplinar. Gutiérrez \& Pérez, (2015).

Para la formación de ciudadanos democráticos, se requiere un conjunto de competencias tales como; participación, cooperación, responsabilidad, tolerancia, solidaridad, habilidades sociales y gestión de conflictos interpersonales, así los estudiantes mediante equipos de trabajo tendrán la capacidad de gestionar democráticamente la resolución de conflictos que alteran la convivencia en la comunidad educativa, adoptando estrategias que permitan la participación de las personas, de tal manera que adquieran un compromiso por dialogar, escuchar, expresar sentimientos y participar en sus propios conflictos, sin desencadenar comportamientos violentos, tanto en el contexto escolar como en el social, Guzmán \& Preciado, (2012).

La educación a lo largo del tiempo ha presentado cambios significativos, que invitan al docente a diseñar e implementar nuevas estrategias pedagógicas para dirigir el proceso de enseñanza con sus estudiantes, una estrategia pedagógica es un conjunto de actividades que se realizan con un orden lógico y coherente en función del cumplimiento de objetivos del plan académico. Es decir, es una planificación que contiene métodos o acciones que permiten al estudiante alcanzar los logros propuestos, mejorar su aprendizaje y facilitar su crecimiento personal (Picardo, Balmore, \& Escobar, 2004).

En ese sentido, pensar la investigación desde las corrientes educativas criticas significa una lucha teórico-práctica por la manera como sus presupuestos sobre el conocimiento, la ciencia, su epistemología, la cultura, lo humano y los grupos sociales enmarcan una acción que durante cuatrocientos años ha sido señalada como objetiva, y que no solo ha construido una forma de ella, sino que también ha ayudado a generar formas de poder que en la sociedad han servido para el control y la gestación de desigualdades, y en estos tiempos, nuevas formas de acumulación y dominación (Ortega, 2009).

Mejía \& Manjarrez, (2010), afirman que la IEP, es una metodología encaminada a fomentar el interés en la población estudiantil por la investigación científica y tecnológica, donde el maestro acompaña a sus educandos en la búsqueda del saber a través de una acción dialógica que incluye el texto, la disciplina y el método científico. El mismo autor señala, que esta herramienta permite llevar la investigación a la esfera educativa y pedagógica, que además genera una reelaboración del campo de éstas, así como de los mecanismos prácticos y de acción que las han constituido.

Por otro lado, la IEP implica el proceso de sistematización de maestros(as) el cual apunta a describir y comprender lo sucedido durante las experiencias pedagógicas, con el fin de explicar los resultados de tales experiencias y las razones por las que se obtuvieron. De esta manera, los maestros estarán en capacidad de hacer reflexiones a los procesos a partir de los roles que cada 
uno cumple, e identificar estrategias para mejorar las experiencias futuras. Todo esto evitara un continuo y deliberado maltrato verbal, físico y/o psicológico en los espacios tanto abiertos como cerrados de aprendizajes, evitando comportamientos como sometimientos, sustos, hostigamientos, intimidad y/o amenazas atentando contra su dignidad humana.

En la implementación de la IEP los contenidos educativos se convierten en problemáticas de posible abordaje y el currículo pasa a estructurarse a partir del surgimiento de una serie de preguntas educativas expuestas por los estudiantes, el avance y desarrollo de los mismos se manifiesta a través del lenguaje, razonamiento y conocimiento que ellos demuestren; ese descubrimiento generalmente no se hace de manera autónoma, es guiado por el docente que se encarga de planificar las estrategias, los ejercicios y los procedimientos para el fin búsqueda. Mejía \& Manjarrez, (2010).

En ese sentido La IEP, tiene como finalidad instaurar bases científicas a los estudiantes en el aula de clases, esta parte de una dinámica metodológica, que busca dar respuesta a los interrogantes de los niños y niñas de la Institución, donde el docente a través de un rol de orientador permita a estos construir bases para comprender el mundo y de esta manera poder interpretarlo y describirlo con un lenguaje científico. En la implementación de la IEP, se parte de la pregunta del sentido común de los niños, niñas y jóvenes para reelaborarla, reconociendo la existencia de saberes comunes y elaborados, llevando la teoría a la práctica para proporcionar un aprendizaje en contexto, (Mariño, 2010).

\section{Metodología}

\section{Diseño}

La metodología utilizada en el estudio es de tipo cualitativo, entendida como una categoría de diseños de investigación que extraen descripciones a partir de observaciones que adoptan la forma de entrevistas, narraciones, notas de campo, grabaciones, transcripciones de audio, vídeo, registros escritos de todo tipo, fotografías o películas y artefactos, Pérez (2012). A su vez se utilizó el modelo de investigación acción que es una metodología de investigación orientada a la práctica educativa.

Para Silva Peña (2012), la investigación acción es una metodología que se ubica en el paradigma crítico-propositivo que, a diferencia del positivista o interpretativo requiere de la participación de los afectados por la preocupación temática estudiada. De este modo, los actores implicados se convierten en los protagonistas del proceso de construcción del conocimiento e intervención sobre la realidad. El rasgo distintivo que adjudica esta autora a la investigaciónacción es que aquellas personas que están afectadas por cambios planificados tienen una responsabilidad primaria en cuanto a decidir acerca de la orientación de una acción críticamente informada que parece susceptible de conducir a una mejora

En esta línea se argumenta que es posible que los docentes (o también otros profesionales prácticos) mejoren sus habilidades o competencias para la ejecución de sus tareas en el campo donde se desempeñan. Dadas las características connaturales de los escenarios escolares, el educador encuentra ahí un espacio propicio para identificar temas y 
problemas inmediatos, comprenderlos, recrearlos y transformarlos. La vida escolar facilita el perfeccionamiento, de capacidades investigadoras para identificar problemas, observar, registrar, interpretar información, experimentar, planear, evaluar y escribir.

\section{Participantes}

La unidad de análisis estuvo conformada por cuarenta (40) estudiantes del grado sexto de la Institución Educativa Buenos Aires, de Aracataca-Magdalena, con edades comprendidas entre los 10 y los 13 años, cabe destacar que todos los participantes cumplieron con los requisitos de participación en el estudio y firmaron el consentimiento informado.

\section{Procedimiento}

Las características principales de este modelo son la transformación y mejora de una realidad escolar, partiendo de la perspectiva de quien posee el problema, implica la colaboración de participantes, requiere la reflexión sistemática en la acción, convirtiendo la práctica en objeto de investigación, formando parte del mismo proceso, esta es realizada por las personas implicadas en la investigación, donde el elemento de formación es esencial en el proceso investigativo. De acuerdo a la naturaleza de la investigación, la metodología se centró en:

a. Selección de un problema general: el problema define al área educativa en la que se centrará la investigación. Al respecto, para este apartado se desarrollaron tres (3) estrategias, entre estas la (1) estrategia diagnóstica de acompañamiento $\mathrm{y}$ entrevistas, dirigidas a directivos, docentes, estudiantes, padres de familia y personal administrativo de la institución educativa. (2) Estrategias de prevención, contempla e incorpora el apoyo y la participación de los aliados estratégicos de la institución educativa; (3) estrategia de intervención inmediata en situaciones de vulneración y riesgo a los estudiantes.

b. Revisión de la bibliografía sobre el problema: se recogen los datos.

c. Formulación del problema, objetivo de la investigación.

d. Determinación del diseño y metodología: el investigador decide de qué sujetos se van a obtener los datos.

e. Recogida de los datos: deben revolverse, además aspectos éticos y legales con respecto a la recogida de datos y su análisis.

f. Análisis de los datos y presentación de los resultados: emplean las representaciones visuales gráficas como tablas estadísticas o diagramas integradores.

g. Interpretación de los resultados y conclusiones respecto al problema: se toman decisiones sobre el formato apropiado del informe.

\section{Resultados}

En la institución se observa un deterioro en la convivencia escolar, esto aunado a comportamientos inadecuados, como por ejemplo, problemas de aceptación, irrespeto a sus semejantes, y falta de tolerancia por sus compañeros; en consecuencia, la distracción aumenta, viéndose afectado el aprendizaje, porque los estudiantes pierden la concentración, 
actitud de aprender y participar activamente en el desarrollo de las actividades pedagógicas, Es evidente entonces, que todo esto influye en todos los entorno de la vida de estudiantes, convirtiéndose en una preocupación de los entes educativos, por ello se hace necesario el fortalecer los valores, para que sean adquiridos de manera eficaz en la formación personal, de tal manera que se reflejen en su relación con sus semejantes y con el entorno, orientados a propiciar una educación integral, democrática, generando espacios de participación activa, mediante una cultura de paz.

Desarrollar este trabajo ha sido de mucho interés, ya que, articula la interrelación de la comunidad educativa con la familia, así mismo, profundiza la investigación, mediante comportamientos respetuosos y representativos que dan un toque especial a una educación educada y participativa en todos los escenarios que son requeridos en los tiempos actuales, en otras palabras,

Se podrá encontrar soluciones pertinentes, que ayudaran a tratar estos tipos de circunstancias, que aplicándolas de forma adecuada podrán cambiar estos malos hábitos en nuestros niños, para que, de esta forma, tanto ellos como sus familiares puedan llevar una mejor convivencia. Al utilizar la investigación como estrategia pedagógica para la convivencia escolar en educación básica, los resultados se orientan a la propuesta de diversas estrategias, (ver Tabla 2).

Como se mencionó anteriormente, el conflicto forma parte de la vida, sin embargo, en determinadas condiciones puede conducir a la violencia, incluso en contextos escolares, en los que, por su naturaleza educativa, resulta más sorprendente Por eso, para mejorar la convivencia educativa y prevenir la violencia es preciso enseñar a resolver conflictos incluidos los relacionados con procedimientos de disciplina de forma constructiva, fomentando el pensamiento, dialogo, negociación, mediación y colaboración, creando así contextos normalizados dentro y fuera del aula de clase a los actores que interviene en dichos procesos, (ver Figura 1).

Tabla 2

Estrategias de formación en la IEP para la convivencia escolar

\begin{tabular}{|c|c|}
\hline Estrategias de formación en la IEP & Convivencia escolar \\
\hline (a) Técnicas para el desarrollo de la tutoría y convivencia. & $\begin{array}{l}\text { La escucha activa. } \\
\text { La observación. } \\
\text { Socio drama/Juego de roles. } \\
\text { Investigación participativa. }\end{array}$ \\
\hline (b) Dinámicas grupales para la tutoría y convivencia. & $\begin{array}{l}\text { Expresión mediante el arte. } \\
\text { Análisis y reflexión. } \\
\text { Aprendizaje cooperativo } \\
\text { Auto evaluación. } \\
\text { De animación y motivación. }\end{array}$ \\
\hline $\begin{array}{l}\text { (c) Instrumentos sugeridos para ser utilizadas en el } \\
\text { trabajo individual y/o grupal. }\end{array}$ & $\begin{array}{l}\text { Ficha acumulativa personal. } \\
\text { Entrevista con el estudiante. } \\
\text { Encuestas. } \\
\text { Anecdotario. } \\
\text { Lista de cotejo. } \\
\text { Ficha de derivación. }\end{array}$ \\
\hline
\end{tabular}

Fuente: elaboración propia (2018). 


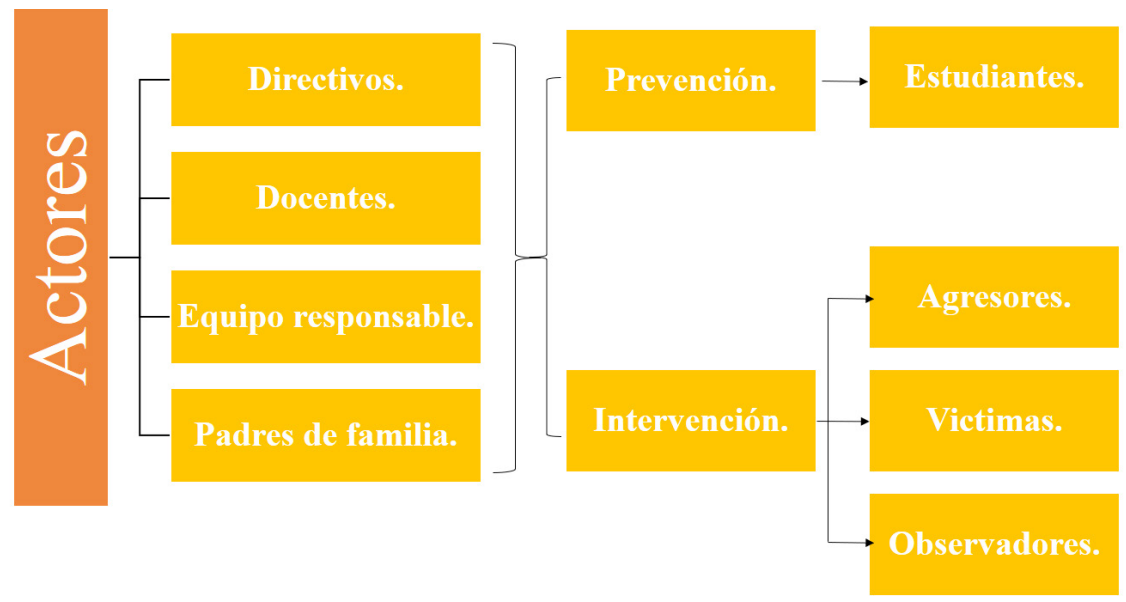

Figura 1. Actores para la convivencia escolar mediante la IEP en la educación básica. Fuente: elaboración propia (2018).

\section{Conclusiones}

La falta de una educación encaminada al fortalecimiento de valores ha dejado en la sociedad actual muchas desventajas, dificultades y espacios vacíos en el aprendizaje de los estudiantes. Estas debilidades responden a muchos factores tales como: insuficiencia de autoridad y función tutelar de los padres, la falta de concienciación por parte de los educandos en crear una sociedad respetuosa tolerante y humanizante.

Utilizar la IEP, para la convivencia escolar en educación básica, posibilita diseñar, articular y poner en funcionamiento un trabajo compartido para elaborarlo y sostenerlo en su aplicación, demanda tiempo para diseñarlo, aplicarlo, probarlo para adecuarlo. Se construye día a día, es decir, que siempre está a prueba, no hay recetas infalibles, sólo se pueden hacer sugerencias y propuestas.

Hay que generar distintos momentos de encuentro y participación entre los actores institucionales, que permitan el diálogo, reflexión, debate. Se debe considerar las características de cada institución educativa, la existencia, adecuación y/o creación de los tiempos institucionales (no es lo mismo contar con la existencia de horas extra clase o tener que hacer la adecuación de los horarios de clase habituales), la disponibilidad y uso de los espacios disponibles.

Es evidente entonces, que hacer de la educación una herramienta de comunicación viva, requiere de un aprendizaje solidario, de critica constructivista y pensamiento abierto, capaz de suscitar transformaciones sociales a través de la participación responsables de todos los involucrados en este proceso, como es la comunidad en general y la institución educativa, en un plano educativo social, y sin duda, constituyen uno de los principales antídotos contra la violencia desde la perspectiva de las llamadas escuelas pacíficas.

Al utilizar la investigación como estrategia pedagógica para la convivencia escolar en educación básica, se derivaron cuatro (4) aspectos necesarios para enriquecer este proceso: (1) sistematizar las normas de convivencia institucional, a partir de las propuestas y sugerencias de los consejos de curso, o resultado de jornadas de convivencia, enmarcándolas 
en la normativa vigente; (2) Intervenir analizando, evaluando y brindando asesoramiento a las autoridades de la escuela o centro escolar en situaciones que afectan la convivencia institucional en general y en situaciones de conflicto institucional en particular; (3) Brindar asesoramiento sobre las sanciones que correspondieren ante faltas y transgresiones; y (4) Participar asesorando o coordinando acciones en situaciones que requieran la aplicación de soluciones alternativas ( mediación y negociación).

Todo lo antes referido, invita a generar distintos momentos de encuentro y participación entre los actores institucionales, que permitan el diálogo, la reflexión, el debate. Quizá sea éste uno de los aspectos más problemáticos, para resolver. Dado que en realidad son pocas las escuelas que cuentan con un tiempo institucional para dedicar al sistema de convivencia, será necesario considerar:

a. Las características de cada institución educativa (cantidad de alumnos, docentes, turnos de funcionamiento), existencia, adecuación y/o creación de los tiempos institucionales (no es lo mismo contar con la existencia de horas extra clase o tener que hacer la adecuación de los horarios de clase habituales),

b. la disponibilidad horaria de los actores en particular para compartir encuentros entre sí.

c. la disponibilidad y uso de los espacios existentes (escuelas que cuentan con salón de actos y/ o patios cubiertos, escuelas que sólo cuentan con algún salón de usos múltiples). Esto incluye también los tiempos en que los mismos pueden ser utilizados; (d) conviene aclarar que tam- bién hay un tiempo que cada institución necesita para ir haciéndose cargo de los movimientos que se han ido generando durante la construcción de su sistema de convivencia escolar; este tiempo no es el que consigna el calendario, sino una consecuencia del estilo o carácter institucional, que surge de la interrelación de los distintos aspectos señalados anteriormente.

\section{Referencias bibliográficas}

Caballero, M. (2010). Convivencia escolar. Un estudio sobre buenas prácticas. Revista Paz y Conflictos. Número 3.

Colombo, G. (2011). Violencia Escolar y Convivencia Escolar: Descubriendo estrategias en la vida cotidiana escolar. Revista Argentina de Sociología, vol. 8-9, núm. 15-16, Consejo de Profesionales en Sociología. Buenos Aires, Argentina.

Gutiérrez, D. \& Pérez, E. (2015). Estrategias para generar la convivencia escolar. Ra Ximhai, vol. 11, núm. 1, enero-junio, 2015, pp. 63-81 Universidad Autónoma Indígena de México. El Fuerte, México.

Guzmán, E \& Preciado, A. (2012). La convivencia escolar: una mirada desde la diversidad cultural. Tesis de Maestría, Universidad de Manizales. Caldas, Manizales.

Herrera Tapias, B. (2013). La constitucionalización de los derechos del consumidor en Colombia: un análisis desde los derechos sociales fundamentales. Civilizar. Ciencias Sociales y Humanas, 13 (25), 33-47.

Ley 1620 de 2013. Guías pedagógicas para la convivencia escolar. Sistema Nacional de Convivencia Escolar. Ministerio de Educación Nacional. 
López, C., Carvajal, C., Soto M., \& Urrea, P. (2013). Factores asociados a la convivencia escolar en adolescentes. Rev. Educación y Educadores vol.16 no.3 Pag.383-410.

Mariño, G. (2010). El diálogo en la educación de jóvenes y adultos. Dos propuestas pedagógicas para implementarlo. En el taller dialógico/la recuperación de experiencias laborales. Bogotá. OEI.

Mejía, M., \& Manjarrés, M. (2010). Las pedagogías fundadas en la investigación. Búsquedas en la reconfiguración de la educación. Revista Internacional Magisterio 42: 16-26. Colombia.

Mendoza, A. (2015). Propuesta de convivencia escolar a través de talleres de prevención de violencia escolar con perspectiva de género. Cultura Educación y Sociedad, 6(2).
Ortega, P., Peñuela, D \& López, D. (2009). Sujetos y prácticas de la pedagogía crítica. Ediciones El Búho. Bogotá.

Ortega, R. (2015). La Convivencia Escolar: qué es y cómo abordarla. Consejería de Educación y Ciencia.

Pérez, G. (2012). Competencias o pensamiento práctico. La construcción de los significados de representación y de acción. Educar por competencias ¿̇qué hay de nuevo? (2aed., pp. 59-102). Madrid, España: Ediciones Morata. Picardo, O., Balmore, R., \& Escobar, J. (2004). Diccionario enciclopédico de ciencias de la educación. San Salvador: El Salvador.

Silva Peña, Ilich (2012). InvestigaciónAcción Realizada por Docentes Chilenos como una vía para su Desarrollo Profesional: Caracterización de dos experiencias. Paradigma 33 (1): 2744. 